\title{
Effective Treatment of a BRAF V600E-Mutant Epithelioid Glioblastoma Patient By Vemurafenib: A Case Report
}

\section{Zhiying Lin}

Southern Medical University Nanfang Hospital

\section{Guozhong Yi}

Southern Medical University Nanfang Hospital

\section{Runwei Yang}

Southern Medical University Nanfang Hospital

\section{Zhiyong Li}

Southern Medical University Nanfang Hospital

\section{Haojie Zheng}

Southern Medical University Nanfang Hospital

\section{Zhu Zhang}

Southern Medical University Nanfang Hospital

\section{Junxiang Peng}

Southern Medical University Nanfang Hospital

Xian Zhang

Southern Medical University Nanfang Hospital

\section{Songtao Qi}

Southern Medical University Nanfang Hospital

Yawei Liu

Southern Medical University Nanfang Hospital

Guanglong Huang ( $\sim$ hgl1020@163.com )

Southern Medical University Nanfang Hospital https://orcid.org/0000-0002-1589-9202

\section{Case report}

Keywords: epithelioid glioblastoma, BRAF inhibitors, whole exome sequencing

Posted Date: November 23rd, 2020

DOI: https://doi.org/10.21203/rs.3.rs-112130/v1 
License: (c) (i) This work is licensed under a Creative Commons Attribution 4.0 International License. Read Full License

Version of Record: A version of this preprint was published at Anti-Cancer Drugs on July 4th, 2021. See the published version at https://doi.org/10.1097/CAD.0000000000001130. 


\section{Abstract}

Background: Novel therapeutic targets in epithelioid glioblastoma (E-GBM) patients are urgently needed. About half of these tumors show the point mutations of the B-Raf proto-oncogene (BRAF). Therefore, this is a target of special interest for this group of patients. Meanwhile, unlike conventional glioblastoma, EGBM lacks specific prognostic markers.

Case presentation: We present a case report of a long-term surviving 37-years-old male patient diagnosed with a BRAF V600E-mutated E-GBM with IDH wild-type. The tumor displayed atypical exophytic growth and an obvious proliferation of vascular endothelial cells. Notably, tumor tissue was found under subarachnoid space. After conventional postoperative treatment options (including radiotherapy and temozolomide chemotherapy) were exhausted, vemurafenib treatment was initiated. The patient maintained clinically stable, and follow-up MRI was consistent with stable disease for the following fifteen months up to now. To identify potential therapeutic targets of E-GBM, we carried out whole exome sequencing analysis and RNA-sequence to study the molecular characteristics of this E-GBM sample. The mutant genes were profiled using glioma RNA-sequencing data from Chinese Glioma Genome Atlas (CGGA) database. We found that CNTNAP3, mTOR, NFATC3, and NOM1 shared by DNA-sequence data and RNA-sequence data were independent prognostic factors. Biological function was associated with phenotypes of focal adhesion and cell cycle. A prognostic risk model was constructed based on the mutant genes for predicting the survival of glioma patients. Survival plot and a nomogram was used to verify that our prognostic risk model is reliable and accurate.

Conclusions: Our findings provided a new sight on the BRAF inhibitor, in combination with which CNTNAP3, mTOR, NFATC3, and NOM1 inhibitors seem to be a valuable salvage treatment option for EGBM.

\section{Background}

Epithelioid glioblastoma (E-GBM) is a rare glioblastoma (GBM) variant newly added to IDH-wild type GBM in the 2016 WHO classification [1]. The tumor is a highly invasive tumor with typical histological features, including closely arranged tumor cells with smooth and round cell boundaries, abundant eosinophilic cytoplasm and the absence of interspersed neuropil [2]. Compared with conventional GBM, E-GBM tends to occur in younger adults and exhibits more aggressive behavior, with a median survival of 5.6 months in children and 6.3 months in adults [3]. The diagnosis of E-GBM is often challenging, as there is still no specific immunohistochemical and molecular markers $[4,5]$. Interestingly, BRAF V600E mutation characteristically occurred in approximately $50 \%$ of all E-GBMs compared with a low frequency in conventional GBM [6].

The BRAF inhibitors targeting the V600E mutation (for example vemurafenib and dabrafenib) represented a breakthrough in the treatment of malignant melanoma. At present, BRAF inhibition is the treatment of 
choice if the V600E mutation is existent $[7,8]$. However, data on clinical use of BRAF inhibitors in patients with glioma are scarce [9-13].

Sequencing techniques have shown great potential in exploring the relationship between genome alterations and tumors [14]. Since the accomplishment of the Human Genome Project (HGP) and the launch of Chinese Glioma Genome Atlas (CGGA), the accumulation of tumor genetic knowledge has increased rapidly in the recent decade. Bioinformatic analysis based on CGGA databases not merely revealed a panorama of tumor-related genome alterations, but also established the basis for comparative studies of tumor relevant types $[15,16]$.

Advances in high-throughput next-generation sequencing technology enable oncologists to decipher the genetic and epigenetic landscapes of tumors [17-21]. Aside from mutations, tumor driver genes may also be altered by transcriptional, methylation, or copy number variation (CNV), which makes the affected genes more difficult to be pinpointed as drivers. A further complicating issue is that mutations in individual gliomas can affect different genes in various combinations. This can alter prognosis and response to therapy $[22,23]$ and poses a challenge to confidently identify genes that are truly collaborating with one another. Therefore, it is important to understand the functional genomic landscapes of glioma.

Despite the use of intensive therapy, the clinical course of E-GBM is aggressive and associated with a high prevalence of hemorrhagic episodes, tumor dissemination in the leptomeninges, and outside the Central Nervous System (CNS) [3]. Here, we present a rare case of an IDH wild-type E-GBM patient with BRAF V600E mutation, in whom clinical and follow-up magnetic resonance images (MRI) stability could be achieved for fifteen months by the treatment of BRAF inhibitor vemurafenib. Furthermore, we provide new insights on the combination of BRAF inhibitors with these mutant genes inhibitors (CNTNAP3, mTOR, NFATC3, and NOM1), which seems to be a valuable treatment option for E-GBM.

\section{Materials And Methods}

\section{Patients and datasets}

The Institutional Review Board of Nanfang Hospital affiliated to Southern Medical University approved the study. We collected 966 gliomas with RNA-sequence (RNA-seq) data and clinical information from Chinese Glioma Genome Atlas (CGGA) database.

\section{Histopathology}

The pathological molecular markers included glial fibrillary acidic protein (GFAP, monoclonal, OriGene, 1:100 dilution), epithelial membrane antigen (EMA, monoclonal, OriGene, 1:100 dilution), 06methylguanine-DNA methyltransferase (MGMT, monoclonal, OriGene, 1:100 dilution), neuronal nuclei (Neu-N, monoclonal,OriGene, 1:100 dilution), oligodendrocyte transcription factor 2 (Oligo-2, monoclonal,OriGene, 1:100 dilution), epidermal growth factor receptor (EGFR, monoclonal, OriGene, 1:100 
dilution), vascular endothelial growth factor (VEGF, polyclonal, OriGene, 1:100 dilution), isocitrate dehydrogenase 1 (IDH1, monoclonal, OriGene, 1:100 dilution), Ki-67 (monoclonal, OriGene, 1:100 dilution), ATRX chromatin remodeler (ATRX, polyclonal, OriGene, 1:100 dilution), BRAF V600E (monoclonal, Roche, 1:100 dilution), H3K27M (polyclonal, OriGene, 1:100 dilution), cytokeratin (CK, monoclonal, OriGene, 1:100 dilution), capicua transcriptional repressor (CIC, monoclonal, OriGene, 1:400 dilution), far upstream element binding protein 1 (FUBP1, monoclonal, OriGene, 1:400 dilution), desmin (monoclonal, OriGene, 1:100 dilution).

\section{Tissue Processing and WES analysis}

Whole exome sequencing (WES) and analysis were carried out at the Genomics Laboratory of GenomicCare Biotechnology (Shanghai, China). For thawed soft tissue, DNA was extracted using the Maxwell RSC Blood DNA Kit (cat\# AS1400, Promega, Madison, WI, USA) on a Maxwell RSC system (cat\# AS4500, Promega). For the FFPE tissue, DNA was extracted using the MagMAX FFPE DNA/RNA Ultra Kit (cat\# A31881, ThermoFisher, Waltham, MA, USA) on a KingFisher Flex system (ThermoFisher). The extracted DNA was sheared using a Covaris L220 sonicator, then the library preparation and capture was done using Tecan EVO 150 (Thermofisher), and sequenced on an Ion S5 sequencer Thermofisher) to generate paired-end reads. After removing adapters and low-quality reads, the reads were aligned to the National Center for Biotechnology Information (NCBI) human genome reference assembly hi19 using the Burrows-Wheeler Aligner alignment algorithm and further processed using the Genome Analysis Toolkit (GATK, version 3.5), including the GATK Realigner Target Creator to identify regions that needed to be realigned. Somatic single-nucleotide variants (SNV), Indel, and CNV were determined using the MuTect/ANNOVAR/dbNSFP31, VarscanIndel, and CNV nator software respectively as reported in Zang et al. [24].

\section{RNA-sequence}

RNA from the FFPE sample was purified using the MagMAX FFPE DNA/RNA Ultra Kit (cat\# A31881, ThermoFisher) on a KingFisher Flex system (ThermoFisher), and used as the template to synthesize cDNA using NEBNext RNA First Strand Synthesis Module (cat\# E7525S, NEB, Waltham, MA, USA) and

NEBNext mRNA Second Strand Synthesis Module (cat\# E6111S, NEB) sequentially. The library preparation, sequencing, and base calling were done similarly as above in the WES section.

\section{Independent prognosis analysis}

The mutant genes shared by DNA-seq and RNA-seq in the sequencing results were selected for further molecular characteristics analysis. With the median expression level of each mutant gene in glioma patients as the boundary, they were divided into high and low expression groups, then Kaplan-Meier method, univariate COX regression analysis, and multivariate COX regression analysis were used for survival analysis and independent prognosis analysis $(P<0.001)$, and the "survival" package and Then, the Wilcoxon rank-sum test was used to analyze the expression of mutant genes in IDH1-mutant (MUT) 
and IDH1-wild type (WT) ( $\mathrm{p}<0.05)$, and the box diagram of clinical correlation analysis was drawn by "ggpubr" package [25, 26].

\section{Bioinformatic analysis}

According to the median expression level of each mutant gene, it was divided into high and low expression groups. With fold change $>2$ and $p<0.05$ as the screening criteria, 966 cases of expression matrix were analyzed, and the differential genes related to mutant genes were screened out. Then use $R$ language "pheatmap" package "limma" package to draw heat map and volcano map of differentially expressed genes. In order to analyze the main function of the mutated genes, the clusterProfiler package [27] of R software was used for GO and KEGG analysis. The 0.05 was set as the cutoff of the p-value. The analysis result was plotted by using ggplot2 package. The analysis results were annotated by Pathview [28] in the R Bioconductor package (https://www.bioconductor.org/).

\section{Construction of Prognostic Risk Model}

Prognostic risk model was to evaluate the accuracy of prognostic models of single variable and for the multivariate prognosis rule by the area under curve (AUC) of the receiver-operating characteristic (ROC) curve. The prognosis risk model of single gene and multi-gene were carried out by using $\mathrm{R}$ language "pROC" package. The multivariate prognostic rules are based on logistic regression models. The ROC curve shows sensitivity and specificity of the binary diagnostic decision for varying cut points based on a single quantitative diagnostic variable or based on a multivariate diagnostic rule.

\section{Identification of a prognostic risk model}

multivariate COX regression model (include Patient's age, gender and WHO grade) was used to evaluate the relationship between each gene and OS of glioma patients by $R$ programming languages. $P<0.05$ is considered statistically significant. Risk characteristics were established according to the regression coefficient weighted gene expression, and the risk scoring formula was constructed as follows:

$$
\text { risk score }=\sum_{n=1}^{I}\left(\operatorname{Exp}_{n} * H R_{n}\right)
$$

In the formula, I is the number of selected genes, $\operatorname{Exp}_{\mathrm{n}}$ is the expression value of each gene, and $\mathrm{HR}_{\mathrm{n}}$ is the multiple Cox regression hazard ration (HR). Glioma patients were divided into low-risk and high-risk groups according to the median risk score, and the performance of prognostic risk characteristics was measured by Kaplan-Meier. The results were visualized in survival curve by R "survival" package. In order to better predict the 1-year, 3-year, and 5-year survival ratio of glioma patients, the prognostic risk model and several clinicopathological factors were included, and a nomogram was established by using the $\mathrm{R}$ rms package based on the results of the multivariate analysis. 


\section{Statistical analysis}

$\mathrm{R}$ language (version 3.5.3) was used for statistical analysis of data: Kaplan-Meier analysis, univariate Cox Return analysis, and multivariate Cox Return analysis were used to identify the mutant genes related to prognosis, Wilcoxon rank-sum test and Kruskal-Wallis $\mathrm{H}$ were used to compare two or more groups for clinical correlation analysis of core genes. The Kaplan-Meier survival curve of each core gene, the forest map of independent prognosis analysis of each core gene, and the box diagram of multiple groups of comparisons were compared. $\mathrm{P}<0.05$ (bilateral) was the difference with statistical significance.

\section{Case Presentation}

A 37-year-old man was admitted to our hospital with sudden dizziness and headache for 5 days (Figure 1). The first magnetic resonance imaging (MRI) showed that there was a lump of abnormal signal shadow with a size of about $4.5 \star 3.0 * 3.0 \mathrm{~cm}$ in the left temporal lobe. Severe edema regions around the lesion were present. To enable a histological diagnosis and treatment decision, tumor resection was performed. The operation was performed from the posterior part of the inferior temporal gyrus, and the old hematoma and grayish red tumor tissue were seen. Extended resection of the tumor was performed along the lateral side of the relative boundary, deep to the temporal horn of the lateral ventricle. After the surgery, the pathological diagnosis was E-GBM. The surgical specimen showed that cancer cells were distributed diffusely, and the cells were arranged closely, which were polygonal, round or triangular, with dense nuclei, visible local nucleoli, partial nuclear deviation, and rich red cytoplasm. Besides, there were obvious cell atypia, visible mitotic cells, obvious proliferation of vascular endothelial cells, vasodilatation and congestion, and flaky necrosis and hemorrhage. Notably, tumor tissue was found in the local subarachnoid space. The immunohistochemical results were listed as the following: Ki-67 (label index: 15\%), GFAP (positive), MGMT (unmethylated), EMA (negative), EGFR (negative), VEGF (positive), ATRX (wild-type), Olig-2 (positive), IDH1 (wild-type), BRAF V600E (mutant), CIC (positive), FUBP1 (positive), H3K27M (negative), Desmin (negative), and CK (negative). Fluorescence in situ hybridization (FISH) detection showed that chromosome (chr) 1p was deleted and 19q was intact. Eight days after operation, the second MRI showed that the left temporal lobe tumor changed after resection, with effusion and a little hematocele in the operation area, and the ependyma of the right lateral ventricle body was slightly thickened.

On the eleventh day after operation, temozolomide (TMZ, Merck Sharp \& Dohme Ltd) $75 \mathrm{mg} / \mathrm{m}^{2}$ was used as neoadjuvant chemotherapy for 17 days. Compared with the second MRI, the third MRI (28 days after operation) showed a new slice-like enhancement in the operation area and tumor recurrence was not ruled out. The fourth MRI of cervical, thoracic and lumbar showed GBM cerebrospinal fluid spread and metastasis, and the dura mater in spinal canal and spinal cord surface was widely thickened and strengthened ( 35 days after operation). Cerebrospinal fluid liquid-based cytology showed that individual malignant tumor cells could be seen ( 35 days after operation). The third and fourth MRI showed that the patient's condition was still progressing after TMZ treatment. Therefore, the patient received 36Gy total central radiotherapy (37 days after operation) for eighteen days, and then locally pushed for 24Gy for 
seven days. During concurrent radiotherapy and chemotherapy, the patient developed third-degree bone marrow suppression. Besides, there are persistent low back pain, a sharp deterioration of mental and dietary conditions, and bed rest and inability to go to the fields. Therefore, the patient stopped radiotherapy and chemotherapy. Since all other conventional treatment options had been exhausted, and to find a target for an experimental salvage therapy, we adopt genomic technology and whole exome sequencing (WES) analysis to study molecular characteristics of this case. The WES sequencing analysis results revealed a V600E mutation of the BRAF kinase. Consecutively, vemurafenib (Roche, BRAF inhibitor) therapy was initiated (960 mg twice daily).

Following vemurafenib treatment, the patient was in stable condition and moves freely. However, seven gastrointestinal reactions (malignant, vomiting, etc.) occurred two weeks after the start of dose treatment. Therefore, we changed the dose of vemurafenib to $480 \mathrm{mg}$, twice daily. So far, patients have been welltolerated and the clinical follow-up was stable. Compared with the fourth MRI, the fifth MRI found that the extensive thickening and enhancement of the endocranium on the spinal canal and spinal cord surface were significantly reduced. WES sequencing analysis and RNA-seq results of formalin-fixed and paraffinembedded (FFPE) tissue revealed nine mutant genes in both DNA-seq data and RNA-seq data (Figure 2A). The protein-protein interaction analysis (PPI) of these nine mutant genes suggested that NFATC3, mTOR, and BRAF interact with each other (Figure 2B). The GBM cohort was divided into a high expression group and a low expression group based on each mutated gene's median expression level, and then univariate and multivariate Cox regression analysis was performed to determine the prognostic value of the nine mutated genes. The results showed that the four mutant genes were regarded as independent prognostic factors in CGGA data. Independent prognostic analysis showed that hazard ration (HR) was 1.2 (95\% confidence interval, Cl: 1.05 1.3) for CNTNAP3, 1.5 (95\% Cl: 1.30 1.546) for mTOR, 1.3 (95\% Cl: 1.19 1.5) for NFATC3 and 1.3 (95\% Cl: 1.19 1.5) for NOM1 Meanwhile, both age and gender are prognostic risk factors (Figure 3A). Thereafter, the Kaplan-Meier method was used to determine the effect of the CNTNAP3, mTOR, NFATC3, and NFATC3 expression on patient's survival. The data showed that patients with high expression of the four mutant genes had significantly shorter overall survival (OS) time than others ( $\mathrm{P}<0.05$, log-rank test) (Figure 3B). Previous reviews have addressed the evidence behind IDH1 as a major prognostic feature for gliomas [29-33]. The gene expression of CNTNAP3, mTOR, NFATC3, and NOM1 in IDH1-wild type (WT) was higher than that in the IDH1-mutant (MUT) group (Figure 3C). The result of CNV analysis revealed deletion of copy number on chromosome 1 and 6 , and amplification of copy number of chromosome 19q and chromosome 21 in this case of E-GBM, as shown in Supplementary Figure 1.

\section{Bioinformatic analysis}

According to the median of each gene expression level, it was divided into high and low expression groups, then taking $|\log 2 \mathrm{FC}|>0.5$ and $p<0.05$ as the screening criteria, analyzing 966 samples of mRNACGGA data. CNTNAP3, mTOR, NFATC3 and NOM1 respectively screened out 2489 related differential genes (down 986, up 1503), 4650 related differential genes (down 93, up 4557), 5930 related differential genes (down 3853, up 2077), and 4143 related differential genes (down 478, up 3665) (Figure 
4A-D). The results of the heat map showed that the most relevant twenty up-regulated genes and twenty down-regulated genes which might be most relevant to each mutant genes (Figure 4A-D).

The case was associated with phenotypes of cell cycle and focal adhesion by GO analysis and KEGG enrichment pathways analysis. GO analysis showed that the related differential genes of CNTNAP3, mTOR, NFATC3, and NOM1 were enriched in biological processes related to cell cycle. For example, the GO terms of cell cycle include regulation of cell cycle G2/M phase transition (G0:1902749, NEDD1, DYNC1H1, NDE1, TAOK1, ATM, FOXN3, DCTN1, PSMD4, CLSPN, PSMB10, PSMC5, etc.), regulation of cell cycle G1/S phase transition (GO:1902806, ADAM17, CNOT6L, RFWD3, GIGYF2, TAF1, STXBP4, KMT2E, EP300, SENP2, etc.), G2/M transition of mitotic cell cycle (GO:0032496, BACH1, TAF2, NEDD1, DYNC1H1, NDE1, FBXL15, TAOK1, ATM, PPM1D, etc.), and regulation of G1/S transition of mitotic cell cycle (G0:2000045, ADAM17, CNOT6L, RFWD3, GIGYF2, KMT2E, EP300, SENP2, ATM, PKD2). Important genes involved in cell cycle in these pathways include ATM, ADAM17, and PKD2. These genes are involved in the cell cycle of GBM [34-36]. GO analysis confirmed that the four mutant genes affected E-GBM through regulation of cell cycle and cell adhesion (Figure 5A-D). Moreover, related differential genes of CNTNAP3, mTOR, NFATC3, and NOM1KEGG were mainly enriched in KEGG enrichment pathways including Glioma, focal adhesion, MAPK signaling pathway, and P13K-Akt signaling pathway (Figure 5A-D). The common KEGG enrichment pathways of the related differential genes of CNTNAP3, mTOR, NFATC3, and NOM1 were the pathways of focal adhesion (hsa04510, HRAS, BRAF, BAD, SHC2, MYL5, PIP5K1A, VCL, MAPK3, GSK3B, MAPK1, etc.), FoxO signaling pathway (hsa04068, SMAD4, HRAS, PRKAA1, STK4, EP300, BRAF, ATM, MAPK3, MAPK11, MAPK1, MAP2K2, STAT3, etc.), and EGFR tyrosine kinase inhibitor resistance (hsa01521, HRAS, BRAF, BAD, SHC2, RPS6KB1, MAPK3, GSK3B, MAPK1, MAP2K2, STAT3, AKT3, etc.). From $\mathrm{GO}$ analysis and KEGG pathway enrichment analysis, it is not difficult to find that the BRAF gene may play an important role in the molecular mechanism of poor prognosis in patients with E-GBM.

\section{Construction of prognostic risk model}

In order to verify the cooperative relationship between CNTNAP3, mTOR, NFATC3, and NOM1, we established a multivariate prognostic risk model. The ROC curve was used to evaluate the efficacy to predict CNTNAP3, mTOR, NFATC3, and NOM1, and their interaction in glioma patients. The areas under curve (AUC) for CNTNAP3, NOM1, NFATC3, mTOR and their interaction were $0.572,0.590,0.623,0.659$ and 0.687 , respectively (Figure $6 \mathrm{~A}$ ).

\section{Constrcution of a nomogram intergating the prognostic risk model and clinicopathological factors}

The prognostic risk model (CNTNAP3, mTOR, NFATC3, and NOM1) were integrated to establish a prognostic risk model. The risk scores were calculated using the formula mentioned in the glioma methods, as follows: risk score $=(1.2 *$ expression level of CNTNAP3 $)+(1.5$ * expression level of mTOR $)$ $+(1.3$ * expression level of NFATC3 $)+(1.2$ * expression level of NOM1), in GBM methods, the risk score $=(1.30 *$ expression level of CDCP1 $)+(1.12$ * expression level of CD44). Glioma patients were respectively divided into low-risk $(n=485)$ and high-risk $(n=485)$ groups according to the median risk 
score. The survival curve showed poorer prognosis in the high-risk group than low-risk group (Figure 6B, P $<0.001)$.

To confirm the prognostic value of risk signature, we constructed a nomogram based on risk signature, and the clinical relevance and prognostic value of age and glioma type (primary glioma, secondary glioma, and recurrent glioma), gender, radiotherapy, TMZ chenmotherapy, and IDH status. The incidence of 1-year, 3-year, and 5-year survival rates can be estimated from the total scores, which are the sum of the scores for each item, as shown in Nomotu (Figure 6C).

\section{Discussion And Conclusions}

In the IDH wild-type and BRAF V600E mutant E-GBM patient treated with vemurafenib as salvage therapy, we achieved clinical stability over fifteen months, which is remarkable at that point of the clinical course after postoperative conventional treatment options were exhausted. Therefore, targeted therapy with BRAF inhibitors may constitute a valuable salvage treatment option. Furthermore, the case showed that when the postoperative conventional treatment options were exhausted in E-GBM patients, especially young patients, it may be helpful to assess whether there is a BRAF mutation.

In malignant melanoma patients with a BRAF V600E mutation, targeted BRAF inhibitors had significantly improved the prognosis $[7,8]$. In contrast, there was little data on the curative effect of patients with brain tumors, particularly GBM. Targeted therapies such as vemurafenib or dabrafenib have been used in a limited number of brain tumor patients with predominantly pleomorphic xanthoastrocytoma, ganglioglioma, and E-GBM [10-13, 37]. Meletath and co-workers reported a case of malignant gliomas arising from ganglioglioma, which produced significant clinical and radiologic responses within 24 months through the use of dabrafenib in combination with tumor-treating fields [10]. Chamberlain [11] treated three adult BRAF V600E mutant ganglioglioma patients with dabrafenib. The median progressionfree survival was seven months (range: 4-10 months). In another case series by the same author [12], similar results were observed in four patients with BRAF V600E-mutated and recurrent pleomorphic xanthoastrocytoma treated with vemurafenib. In a recently published case series, Carry [37] reported a secondary E-GBM patient who survived for 16 months after dabrafenib treatment initiation. The E-GBM patient was arising from an anaplastic astrocytoma after ten years of first-line and second-line treatment. Liangliang successfully treated a BRAF V600E-mutated extraneural metastatic anaplastic oligoastrocytoma with vemurafenib and everolimus (mTOR inhibitor) [38].

Up to now, our patients still show clinical stability over fifteen months with vemurafenib. The survival rate of our patients is comparable to or even longer than that of other patients with BRAF V600E mutation [6, $39,40]$. A recent meta-analysis in glioma patients demonstrated an improved overall survival (hazard ratio: 0.6) if a BRAF mutation was present [41]. That meta-analysis also revealed that a BRAF V600E mutation improved the survival of children and young adults with gliomas but did not have prognostic value in older adults. On the other hand, children with a newly diagnosed epithelioid glioblastoma suffer from an overall poor prognosis, independent of a BRAF V600E mutation [3]. However, the possible 
molecular mechanisms of E-GBM patients are still unclear. Therefore we obtained nine share mutant genes in DNA-seq data by WES analysis and RNA-seq data. We analyze the expression and prognostic correlation of these nine genes in CGGA RNA sequence data. Among them, CNTNAP3, mTOR, NFATC3, and NOM1 are independent prognostic risk factors. The results of multivariate Cox regression analyses showed that patients with high expression of the four mutant genes had significantly shorter OS than others.

Mammalian target of rapamycin (mTOR) represents the serine/ threonine protein kinase and plays a critical role in response to various signal stimuli from mitogen, cytokine, nutritional status, and cellular energy level. Its major functions and activities are under the regulation of PI3K/ protein kinase B (PKB) signal pathway, and mTOR exerts its signal transduction function at downstream of PI3K/AKT pathway. Previous work showed that abnormality of mTOR expression or functional activity resulted in occurrence, progression, and resistance acquirement in multiple tumors [42-45].

The Nuclear Factor of Activated T cells (NFAT) family includes four classic members: c1, c2, c3, and c4, which were firstly described in immune cells $[46,47]$. Katia reported that NFATc3 controls tumor growth by regulating proliferation and migration of human astroglioma cells [48]. The research of contact in associated protein family member 3 (CNTNAP3) and nuclear mif4g domain-containing protein 1 (NOM1) in glioma has not been found yet. Then through Wilcoxon rank-sum test, we found that the gene expression of CNTNAP3, mTOR, NFATC3, and NOM1 in IDH1-WT was higher than that in the IDH1-MUT group. The GO analysis and KEGG enrichment pathway showed that the case was associated with phenotypes of cell cycle and focal adhesion. Future studies with more cases of E-GBM patients are warranted to confirm these preliminary but promising results. Furthermore, it has been demonstrated that vemurafenib has a limited ability to penetrate the blood-brain barrier [49]. Thus, a future effort should be directed to develop new BRAF inhibitors that can effectively penetrate the blood-brain barrier. Patients with BRAF 600E-mutated E-GBM may be accompanied by mutations of CNTNAP3, mTOR, NFATC3, and NOM1, which may affect the prognosis of glioma patients through the regulation of cell cycle and focal adhesion. Therefore, for E-GBM, when screening BRAF, it should also suggest screening these genes, since in these cases targeted therapy with BRAF inhibitor combined with CNTNAP3, mTOR, NFATC3, and NOM1 inhibitors seems to be a valuable salvage treatment option.

\section{Abbreviations}

E-GBM (Epithelioid glioblastoma ); WES (whole exome sequencing); WHO (World Health Organization); HGP (Human Genome Project); CGGA (Chinese Glioma Genome Atlas); MRI (magnetic resonance images); GBM (glioblastoma); KEGG (Kyoto Encyclopedia of Genes and Genomes); GO (Gene Ontology); CNS (Central Nervous System); NCBI (National Center for Biotechnology Information); SNV (Somatic single-nucleotide variants); CNV (copy number variation); IDH1(isocitrate dehydrogenase 1); IDH-wt (IDHwidetype);

\section{Declarations}


Ethics approval and consent to participate

A written consent was obtained from a patient. The Institutional Review Board of Nanfang Hospital affiliated to Southern Medical University approved the study.

\section{Consent for publication}

Written informed consent was obtained from the patients for publication of this case reports. All the authors listed have approved the enclosed manuscript.

\section{Availability of data and material}

The data analyzed in this study can be accessed by sending a request to the corresponding author.

\section{Competing interests}

The authors declare that they have no competing interests.

\section{Funding}

This study was supported by the Guangdong Science and Technology Department (2016A040403053).

\section{Authors' contributions}

Conception and design of the work: YWL and GLH. Acquisition, analysis and interpretation of data: ZYL, GZY, RWY, ZYL, HJZ, STQ, ZZ, JXP, XAZ, YWL and GLH. Drafting of the manuscript: ZYL, GZY, RWY, and GLH. Critical revision for important intellectual content: ZYL, GZY, RWY, ZYL, HJZ, STQ, ZZ, JXP, XAZ, YWL and GLH. All authors approved the final version of this manuscript.

\section{Acknowledgements}

A particular acknowledgment to the medical staff involved in the study.

\section{Author information}

\section{Affiliations}

Department of Neurosurgery, Nanfang Hospital, Southern Medical University, Guangzhou 510515, Guangdong, China

Zhiying Lin; Guozhong Yi; Runwei Yang; Zhiyong Li; Haojie Zheng; Zhu Zhang; Junxiang Peng; Xian Zhang; Songtao Qi; Yawei Liu; Guanglong Huang

The Laboratory for Precision Neurosurgery, Nanfang Hospital, Southern Medical University, Guangzhou 510515, Guangdong, China 
Zhiying Lin; Guozhong Yi; Runwei Yang; Zhiyong Li; Haojie Zheng; Zhu Zhang; Junxiang Peng; Xian Zhang; Songtao Qi; Yawei Liu; Guanglong Huang

Nanfang Glioma Center, Guangzhou 510515, Guangdong, China

Guozhong Yi; Zhiyong Li; Xian Zhang; Songtao Qi; Guanglong Huang

Corresponding authors

Correspondence to Guanglong Huang,

\section{References}

1. Louis DN, Perry A, Reifenberger G, von Deimling A, Figarella-Branger D, Cavenee WK, Ohgaki H, Wiestler OD, Kleihues P, Ellison DW: The 2016 World Health Organization Classification of Tumors of the Central Nervous System: a summary. Acta Neuropathol 2016, 131:803-820.

2. Villa C, Miquel C, Mosses D, Bernier M, Di Stefano AL: The 2016 World Health Organization classification of tumours of the central nervous system. Presse Med 2018, 47:e187-e200.

3. Broniscer A, Tatevossian RG, Sabin ND, Klimo P, Jr., Dalton J, Lee R, Gajjar A, Ellison DW: Clinical, radiological, histological and molecular characteristics of paediatric epithelioid glioblastoma. Neuropathol Appl Neurobiol 2014, 40:327-336.

4. Alexandrescu S, Korshunov A, Lai SH, Dabiri S, Patil S, Li R, Shih CS, Bonnin JM, Baker JA, Du E, et al: Epithelioid Glioblastomas and Anaplastic Epithelioid Pleomorphic Xanthoastrocytomas-Same Entity or First Cousins? Brain Pathol 2016, 26:215-223.

5. Tanaka S, Nakada M, Nobusawa S, Suzuki SO, Sabit H, Miyashita K, Hayashi Y: Epithelioid glioblastoma arising from pleomorphic xanthoastrocytoma with the BRAF V600E mutation. Brain Tumor Pathol 2014, 31:172-176.

6. Kleinschmidt-DeMasters BK, Aisner DL, Birks DK, Foreman NK: Epithelioid GBMs show a high percentage of BRAF V600E mutation. Am J Surg Patho/2013, 37:685-698.

7. Chapman PB, Robert C, Larkin J, Haanen JB, Ribas A, Hogg D, Hamid O, Ascierto PA, Testori A, Lorigan PC, et al: Vemurafenib in patients with BRAFV600 mutation-positive metastatic melanoma: final overall survival results of the randomized BRIM-3 study. Ann Oncol 2017, 28:2581-2587.

8. Hauschild A, Grob J-J, Demidov LV, Jouary T, Gutzmer R, Millward M, Rutkowski P, Blank CU, Miller WH, Kaempgen E, et al: Dabrafenib in BRAF-mutated metastatic melanoma: a multicentre, open-label, phase 3 randomised controlled trial. The Lancet 2012, 380:358-365.

9. Burger MC, Ronellenfitsch MW, Lorenz NI, Wagner M, Voss M, Capper D, Tzaridis T, Herrlinger U, Steinbach JP, Stoffels G, et al: Dabrafenib in patients with recurrent, BRAF V600E mutated malignant glioma and leptomeningeal disease. Oncol Rep 2017, 38:3291-3296.

10. Meletath SK, Pavlick D, Brennan T, Hamilton R, Chmielecki J, Elvin JA, Palma N, Ross JS, Miller VA, Stephens PJ, et al: Personalized Treatment for a Patient With a BRAF V600E Mutation Using 
Dabrafenib and a Tumor Treatment Fields Device in a High-Grade Glioma Arising From Ganglioglioma. J Natl Compr Canc Netw 2016, 14:1345-1350.

11. Chamberlain MC: Recurrent ganglioglioma in adults treated with BRAF inhibitors. CNS Onco/2016, 5:27-29.

12. Chamberlain MC: Salvage therapy with BRAF inhibitors for recurrent pleomorphic xanthoastrocytoma: a retrospective case series. J Neurooncol 2013, 114:237-240.

13. Lee EQ, Ruland S, LeBoeuf NR, Wen PY, Santagata S: Successful Treatment of a Progressive BRAF V600E-Mutated Anaplastic Pleomorphic Xanthoastrocytoma With Vemurafenib Monotherapy. J Clin Oncol 2016, 34:e87-89.

14. Bailey M, Tokheim C, Porta-Pardo E, Sengupta S, Bertrand D, Weerasinghe A, Colaprico A, Wendl M, Kim J, Reardon B, et al: Comprehensive Characterization of Cancer Driver Genes and Mutations. Cell 2018, 173:371-385.e318.

15. Feng X, Zhang L, Ke S, Liu T, Hao L, Zhao P, Tu W, Cang S: High expression of GPNMB indicates an unfavorable prognosis in glioma: Combination of data from the GEO and CGGA databases and validation in tissue microarray. Oncol Lett 2020, 20:2356-2368.

16. Liu W, Xu Z, Zhou J, Xing S, Li Z, Gao X, Feng S, Xiao Y: High Levels of HIST1H2BK in Low-Grade Glioma Predicts Poor Prognosis: A Study Using CGGA and TCGA Data. Front Oncol 2020, 10:627.

17. Capper D, Jones DTW, Sill M, Hovestadt V, Schrimpf D, Sturm D, Koelsche C, Sahm F, Chavez L, Reuss DE, et al: DNA methylation-based classification of central nervous system tumours. Nature 2018, 555:469-474.

18. Parsons DW, Jones S, Zhang X, Lin JC, Leary RJ, Angenendt P, Mankoo P, Carter H, Siu IM, Gallia GL, et al: An integrated genomic analysis of human glioblastoma multiforme. Science 2008, 321:18071812.

19. Cancer Genome Atlas Research N: Comprehensive genomic characterization defines human glioblastoma genes and core pathways. Nature 2008, 455:1061-1068.

20. Bai H, Harmanci AS, Erson-Omay EZ, Li J, Coskun S, Simon M, Krischek B, Ozduman K, Omay SB, Sorensen EA, et al: Integrated genomic characterization of IDH1-mutant glioma malignant progression. Nat Genet 2016, 48:59-66.

21. Ceccarelli M, Barthel FP, Malta TM, Sabedot TS, Salama SR, Murray BA, Morozova O, Newton Y, Radenbaugh A, Pagnotta SM, et al: Molecular Profiling Reveals Biologically Discrete Subsets and Pathways of Progression in Diffuse Glioma. Cell 2016, 164:550-563.

22. Hanahan D, Weinberg RA: Hallmarks of cancer: the next generation. Cel/ 2011, 144:646-674.

23. Krex D, Klink B, Hartmann C, von Deimling A, Pietsch T, Simon M, Sabel M, Steinbach JP, Heese O, Reifenberger G, et al: Long-term survival with glioblastoma multiforme. Brain 2007, 130:2596-2606.

24. Zang YS, Dai C, Xu X, Cai X, Wang G, Wei J, Wu A, Sun W, Jiao S, Xu Q: Comprehensive analysis of potential immunotherapy genomic biomarkers in 1000 Chinese patients with cancer. Cancer Med 2019, 8:4699-4708. 
25. Liu YQ, Chai RC, Wang YZ, Wang Z, Liu X, Wu F, Jiang T: Amino acid metabolism-related gene expression-based risk signature can better predict overall survival for glioma. Cancer Sci 2019 , 110:321-333.

26. Hughey JJ, Butte AJ: Robust meta-analysis of gene expression using the elastic net. Nucleic Acids Res 2015, 43:e79.

27. Yu G, Wang LG, Han Y, He QY: clusterProfiler: an R package for comparing biological themes among gene clusters. OMICS 2012, 16:284-287.

28. Luo W, Pant G, Bhavnasi YK, Blanchard SG, Jr., Brouwer C: Pathview Web: user friendly pathway visualization and data integration. Nucleic Acids Res 2017, 45:W501-W508.

29. Yan H, Parsons DW, Jin G, McLendon R, Rasheed BA, Yuan W, Kos I, Batinic-Haberle I, Jones S, Riggins GJ, et al: IDH1 and IDH2 mutations in gliomas. N Engl J Med 2009, 360:765-773.

30. Watanabe T, Nobusawa S, Kleihues P, Ohgaki H: IDH1 mutations are early events in the development of astrocytomas and oligodendrogliomas. Am J Pathol 2009, 174:1149-1153.

31. Nobusawa S, Watanabe T, Kleihues P, Ohgaki H: IDH1 mutations as molecular signature and predictive factor of secondary glioblastomas. Clin Cancer Res 2009, 15:6002-6007.

32. Karsy M, Guan J, Cohen AL, Jensen RL, Colman H: New Molecular Considerations for Glioma: IDH, ATRX, BRAF, TERT, H3 K27M. Curr Neurol Neurosci Rep 2017, 17:19.

33. Bleeker FE, Atai NA, Lamba S, Jonker A, Rijkeboer D, Bosch KS, Tigchelaar W, Troost D, Vandertop WP, Bardelli A, Van Noorden CJF: The prognostic IDH1 R132 mutation is associated with reduced NADP+dependent IDH activity in glioblastoma. Acta Neuropathologica 2010, 119:487-494.

34. Zhou X, Xue P, Yang M, Shi H, Lu D, Wang Z, Shi Q, Hu J, Xie S, Zhan W, Yu R: Protein kinase D2 promotes the proliferation of glioma cells by regulating Golgi phosphoprotein 3. Cancer Lett 2014, 355:121-129.

35. Chen J, Chen X, Wang F, Gao H, Hu W: Dihydroartemisinin suppresses glioma proliferation and invasion via inhibition of the ADAM17 pathway. Neurol Sci 2015, 36:435-440.

36. Ferreira WAS, Burbano RR, do OPC, Harada ML, do Nascimento Borges B, de Oliveira EHC: Pisosterol Induces G2/M Cell Cycle Arrest and Apoptosis via the ATM/ATR Signaling Pathway in Human Glioma Cells. Anticancer Agents Med Chem 2020, 20:734-750.

37. Ceccon G, Werner JM, DunkI V, Tscherpel C, Stoffels G, Brunn A, Deckert M, Fink GR, Galldiks N: Dabrafenib Treatment in a Patient with an Epithelioid Glioblastoma and BRAF V600E Mutation. Int $J$ Mol Sci 2018, 19.

38. Shi L, Zou Z, Ding Q, Liu Q, Zhou H, Hong X, Peng G: Successful treatment of a BRAF V600E-mutant extracranial metastatic anaplastic oligoastrocytoma with vemurafenib and everolimus. Cancer Biol Ther 2019, 20:431-434.

39. Chi AS, Batchelor TT, Yang D, Dias-Santagata D, Borger DR, Ellisen LW, lafrate AJ, Louis DN: BRAF V600E mutation identifies a subset of low-grade diffusely infiltrating gliomas in adults. $J$ Clin Oncol 2013, 31:e233-236. 
40. Dahiya S, Emnett RJ, Haydon DH, Leonard JR, Phillips JJ, Perry A, Gutmann DH: BRAF-V600E mutation in pediatric and adult glioblastoma. Neuro Oncol 2014, 16:318-319.

41. Vuong H, Altibi A, Duong U, Ngo H, Pham T, Fung K, Hassell LJMn: BRAF Mutation is Associated with an Improved Survival in Glioma-a Systematic Review and Meta-analysis. 2018, 55:3718-3724.

42. Tsubamoto H, Inoue K, Sakata K, Ueda T, Takeyama R, Shibahara H, Sonoda T: Itraconazole Inhibits AKT/mTOR Signaling and Proliferation in Endometrial Cancer Cells. Anticancer Res 2017, 37:515519.

43. Pinto-Leite R, Arantes-Rodrigues R, Sousa N, Oliveira PA, Santos L: mTOR inhibitors in urinary bladder cancer. Tumour Biol 2016, 37:11541-11551.

44. Guerrero-Zotano A, Mayer IA, Arteaga CL: PI3KJAKT/mTOR: role in breast cancer progression, drug resistance, and treatment. Cancer Metastasis Rev 2016, 35:515-524.

45. Su PF, Song SQ: Regulation of mTOR by miR-107 to facilitate glioma cell apoptosis and to enhance cisplatin sensitivity. Eur Rev Med Pharmacol Sci 2018, 22:6864-6872.

46. Jain J, Miner Z, Rao A: Analysis of the preexisting and nuclear forms of nuclear factor of activated T cells. J Immunol 1993, 151:837-848.

47. Martinez-Martinez S, Redondo JM: Inhibitors of the calcineurin/NFAT pathway. Curr Med Chem 2004, 11:997-1007.

48. Urso K, Fernandez A, Velasco P, Cotrina J, de Andres B, Sanchez-Gomez P, Hernandez-Lain A, Hortelano S, Redondo JM, Cano E: NFATc3 controls tumour growth by regulating proliferation and migration of human astroglioma cells. Sci Rep 2019, 9:9361.

49. Falchook GS, Long GV, Kurzrock R, Kim KB, Arkenau TH, Brown MP, Hamid O, Infante JR, Millward M, Pavlick AC, et al: Dabrafenib in patients with melanoma, untreated brain metastases, and other solid tumours: a phase 1 doseescalation trial. Lancet 2012, 379:1893-1901.

\section{Figures}


March 12th, 2019

Tumor relapse in the

left temporal lobe
March 15th, 2019

Tumor tissue was found in

local subarachnoid space
April 9th, 2019

The thirdly MR
April 18th, 2019

Radiotherapy and

TMZ chemotherapy

(25 days)

March 9th, 2019

Admitted to hospital with

sudden headache and

dizziness for 5 days
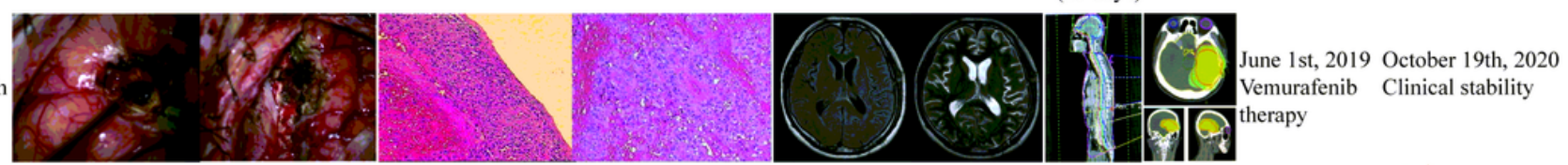

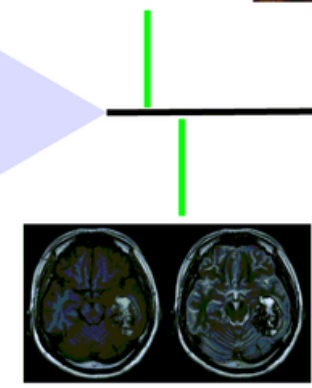

March 10th, 2019 The first MRIt

suggested space

occupying lesions in

the left temporal lobe

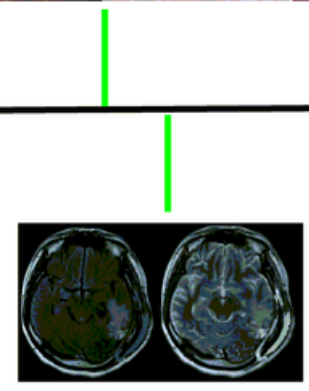

March 20th, 2019

The second MRI

and diagnosis of

E-GBM

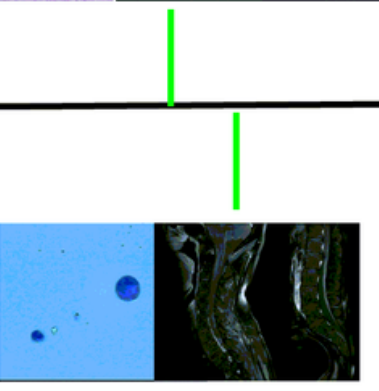

April 16th, 2019

Liquid-based cytology of cerebrospinal fluid and the fourth

MRI of cervical, thoracic and

lumbar vertebrae

\section{Figure 1}

Overview of the patient's course of disease and therapeutic regimens.

\section{March 12th, 2019 \\ Tumor relapse in the \\ left temporal lobe}

\begin{abstract}
March 15th, 2019
Tumor tissue was found in

local subarachnoid space
\end{abstract}

April 9th, 2019
The thirdly MR
April 18th, 2019

Radiotherapy and

TMZ chemotherapy

( 25 days)

\section{March 9th, 2019}

Admitted to hospital with

sudden headache and

dizziness for 5 days
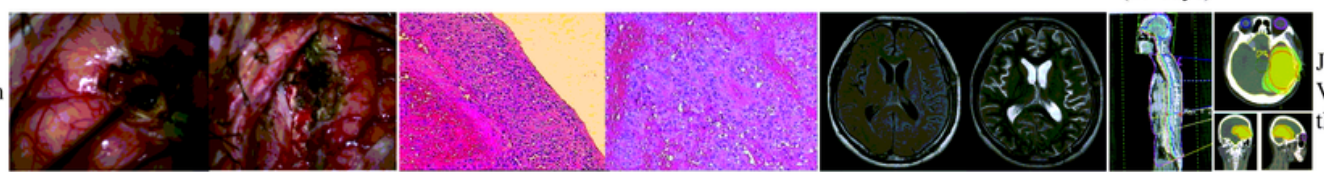

une 1st, 2019 October 19th, 2020

Vemurafenib Clinical stability

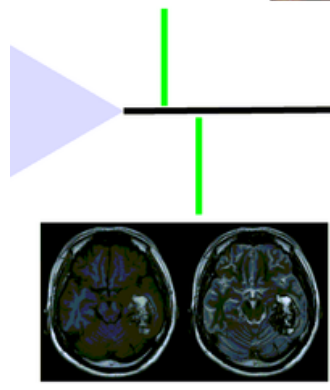

March 10th, 2019

The first MRIt

suggested space

occupying lesions in

the left temporal lobe

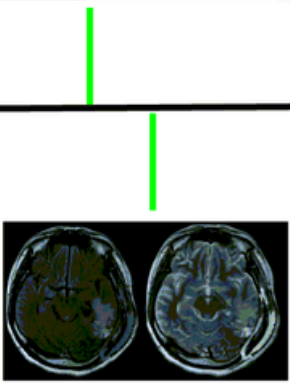

March 20th, 2019

The second MRI

and diagnosis of

E-GBM

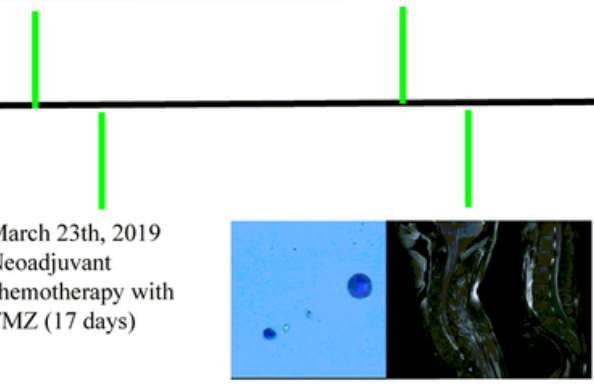

April 16th, 2019

Liquid-based cytology of

cerebrospinal fluid and the fourth

MRI of cervical, thoracic and

lumbar vertebrae

Figure 1

Overview of the patient's course of disease and therapeutic regimens. 


\section{A}

\section{DNA-seq data}
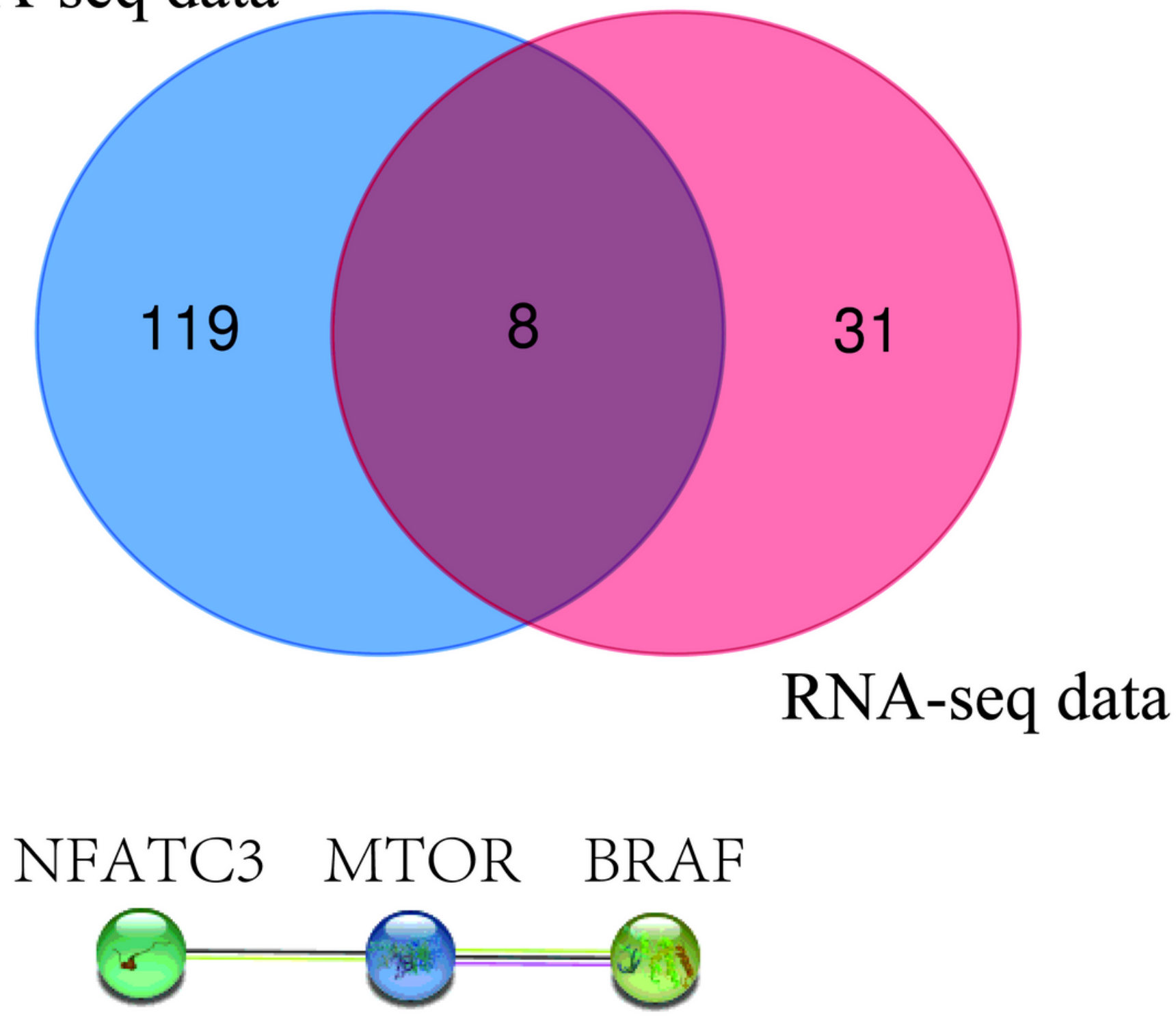

Figure 2

Venn diagram (A) shows the common mutant genes in both DNA-seq data and RNA-seq data. The PPI network $(B)$ of the common mutant genes in both DNA-seq data and RNA-seq data. 


\section{A}

\section{DNA-seq data}
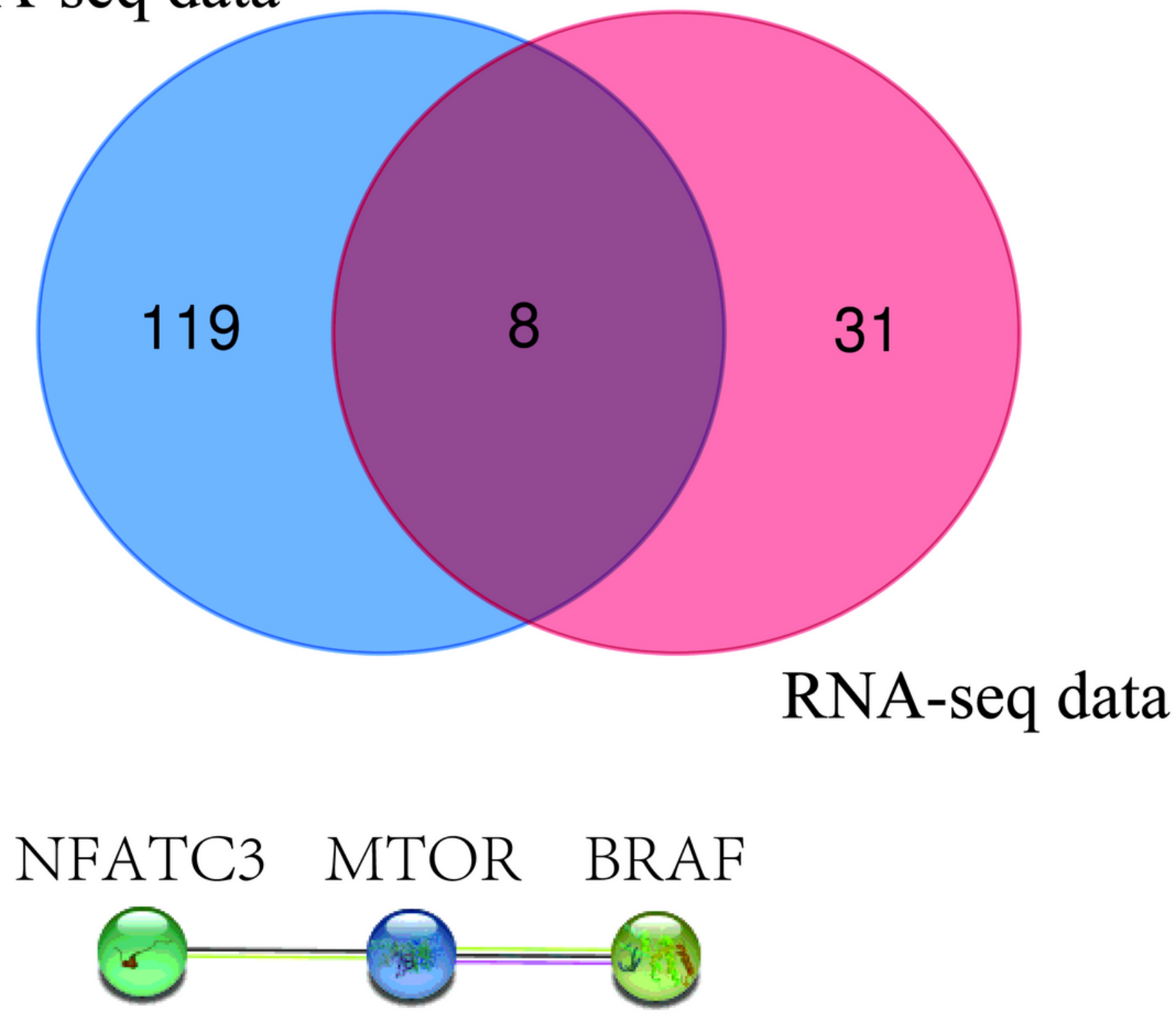

Figure 2

Venn diagram (A) shows the common mutant genes in both DNA-seq data and RNA-seq data. The PPI network $(B)$ of the common mutant genes in both DNA-seq data and RNA-seq data. 

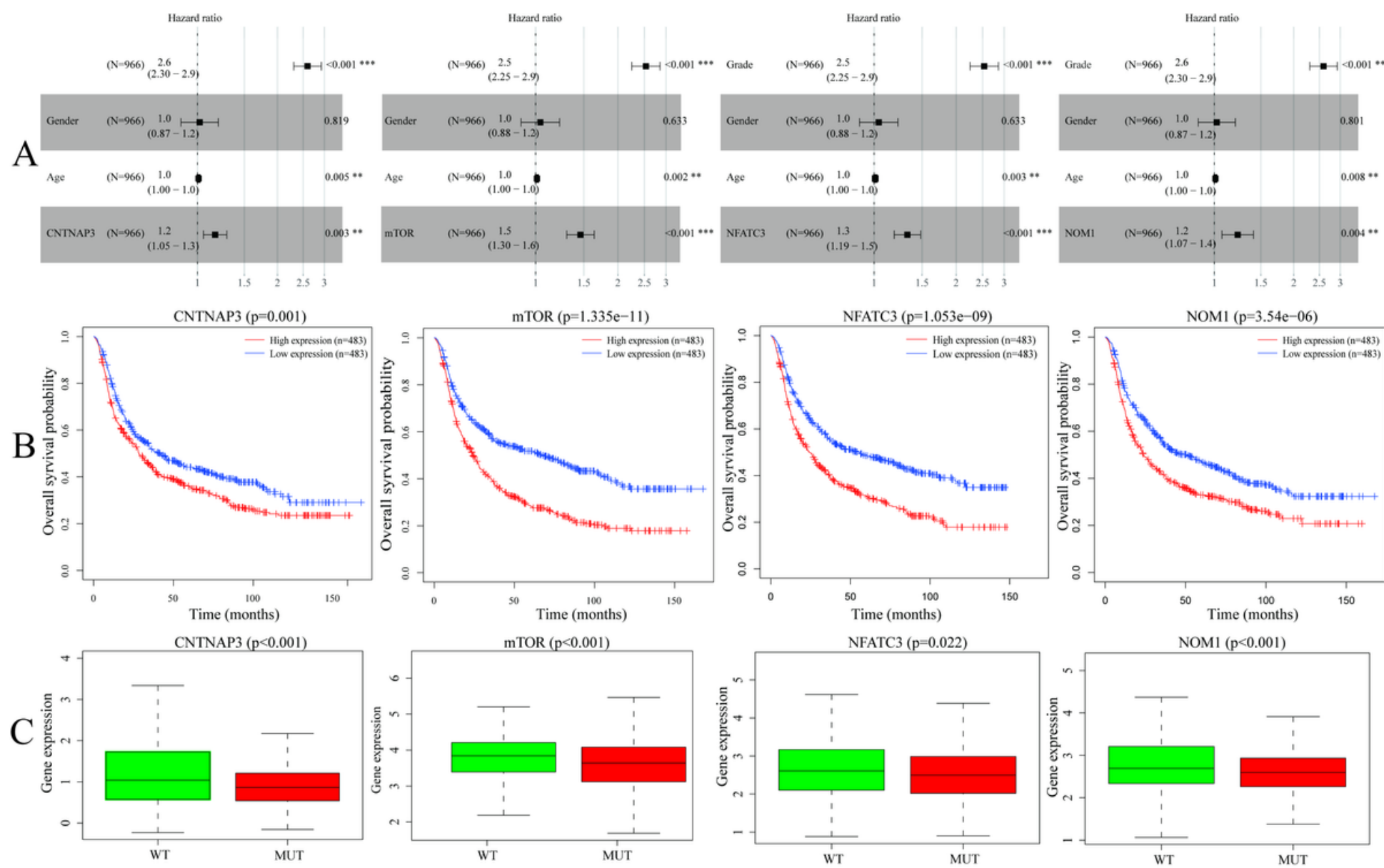

Figure 3

Forest plot (A), survival plot (B) and box plot (C) of the four common mutated genes (from left to right: CNTNAP3, mTOR, NFATC3, and NOM1). 

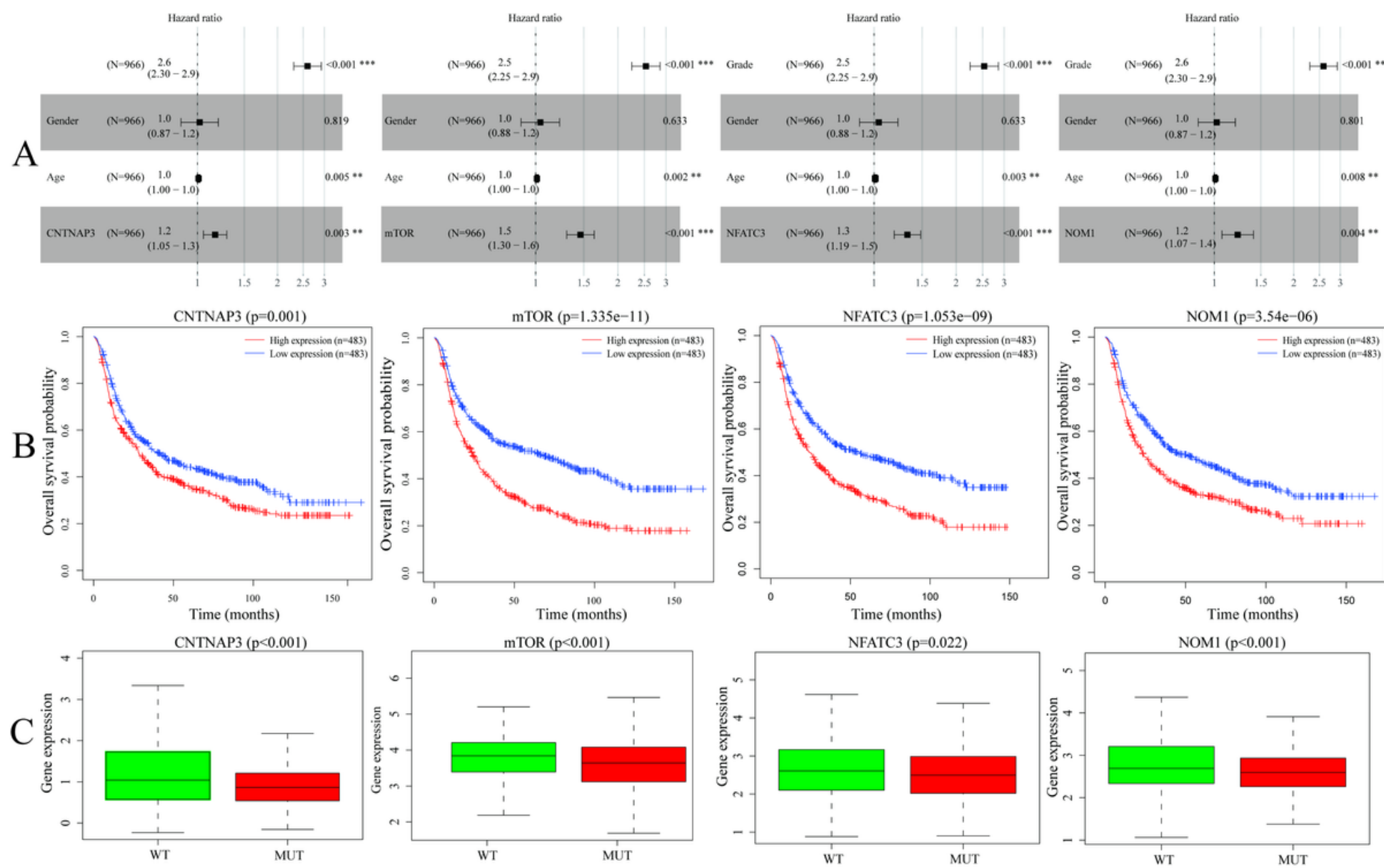

Figure 3

Forest plot (A), survival plot (B) and box plot (C) of the four common mutated genes (from left to right: CNTNAP3, mTOR, NFATC3, and NOM1). 


\section{CNTNAP3 high CNTNAP3 low}

A
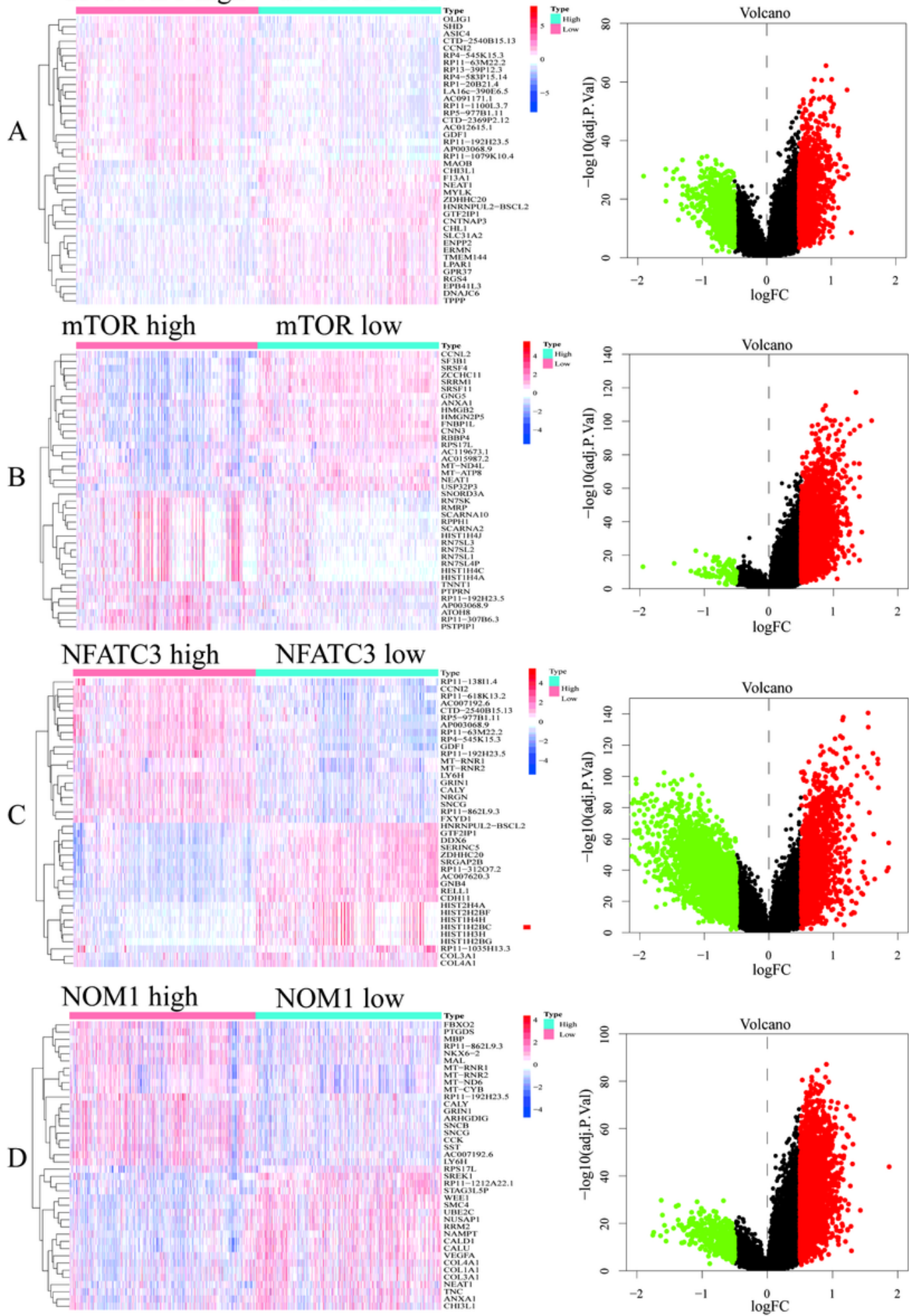

Figure 4

Heat map and volcano map of differential genes related to CNTNAP3 (A), mTOR (B), NFATC3 (C), and NOM1 (D). 

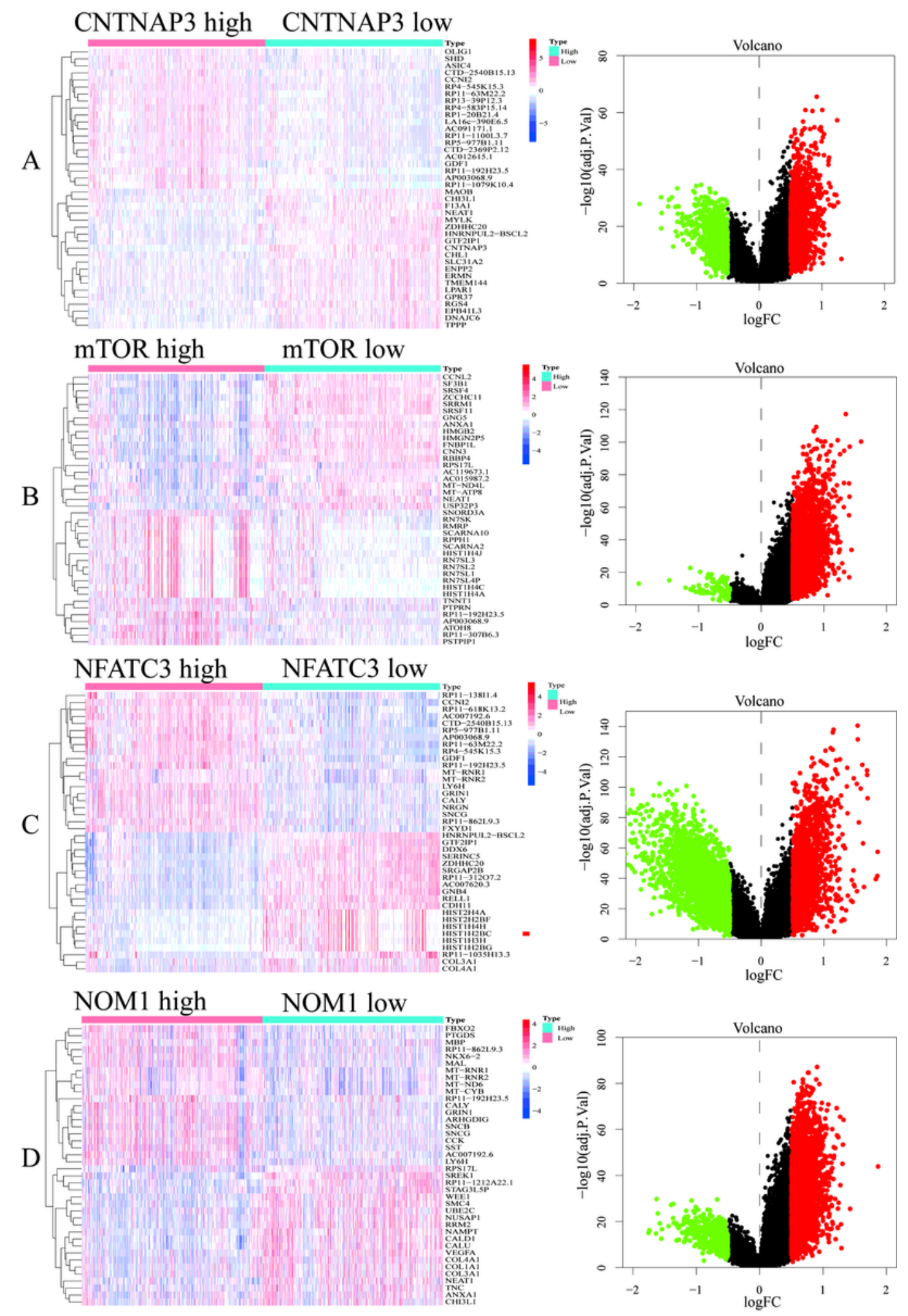

Figure 4

Heat map and volcano map of differential genes related to CNTNAP3 (A), mTOR (B), NFATC3 (C), and NOM1 (D). 


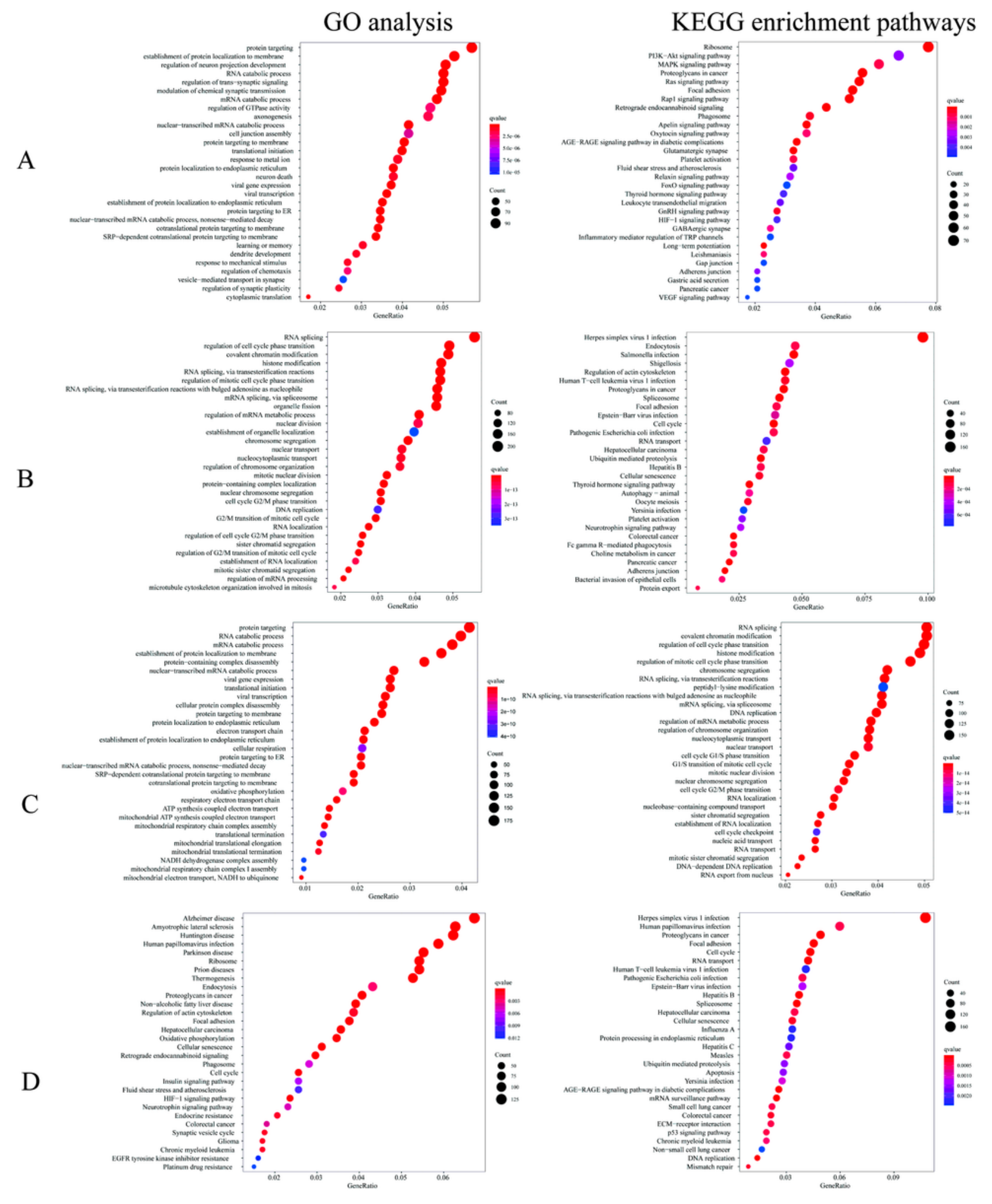

Figure 5

GO and KEGG enrichment pathways analysis of differential genes related to CNTNAP3 (A), mTOR (B), NFATC3 (C), and NOM1 (D). The color change of the circle represents the significant degree of the enriched genes in the pathways. 


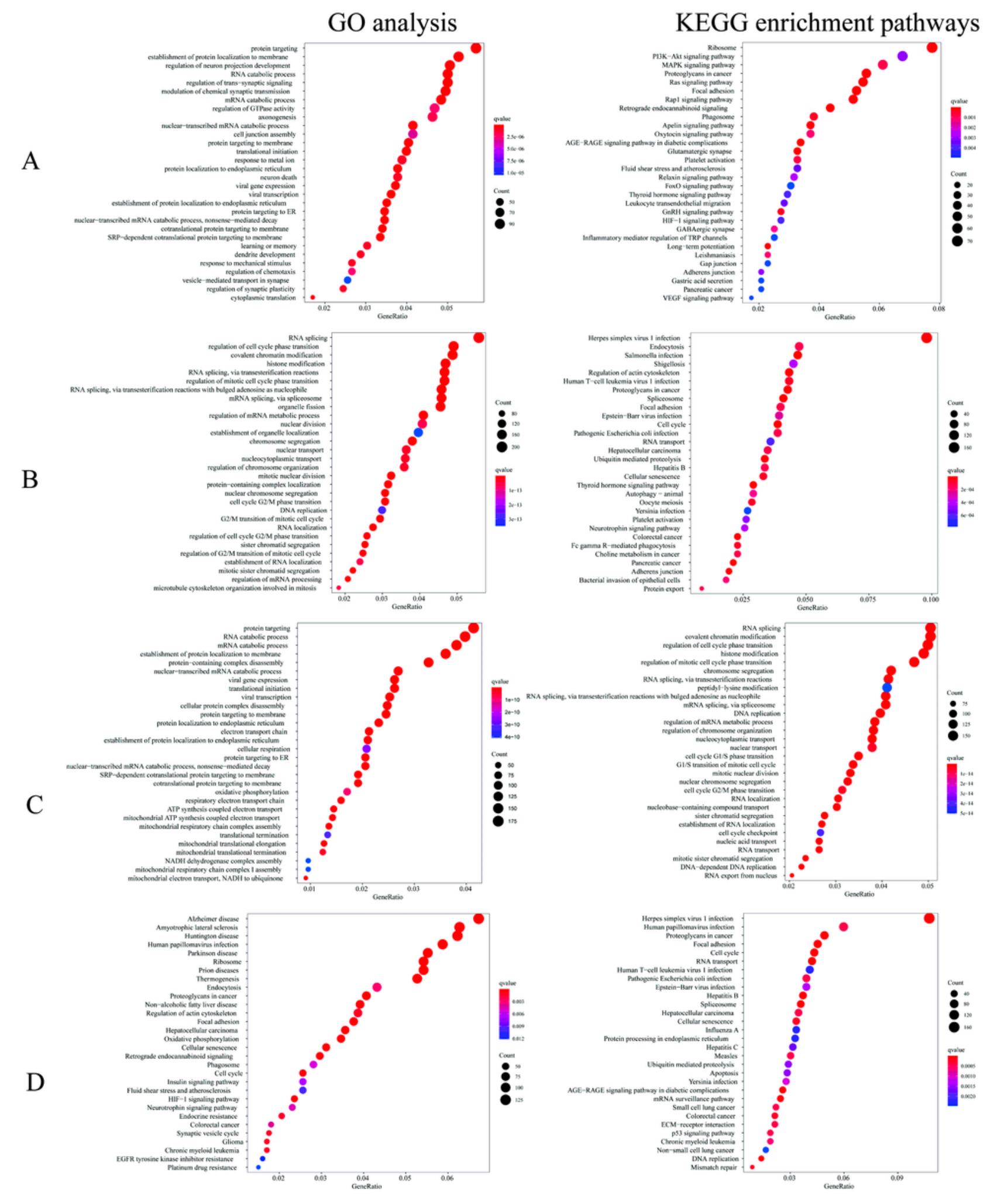

Figure 5

GO and KEGG enrichment pathways analysis of differential genes related to CNTNAP3 (A), mTOR (B), NFATC3 (C), and NOM1 (D). The color change of the circle represents the significant degree of the enriched genes in the pathways. 


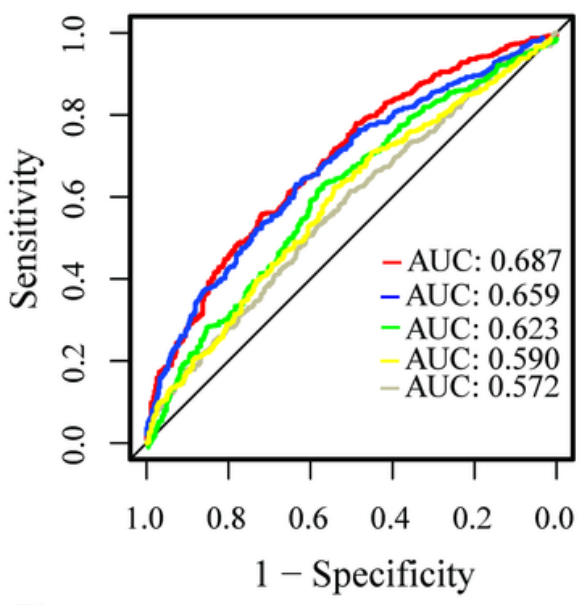

B

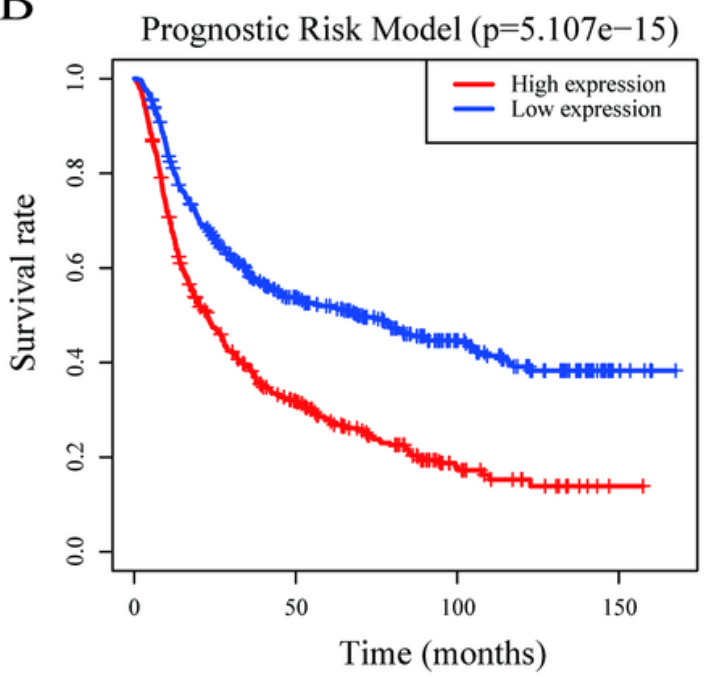

Points

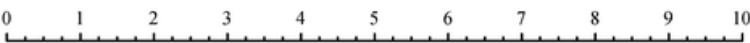

Age

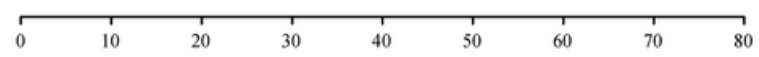

Risk Score

Glioma type
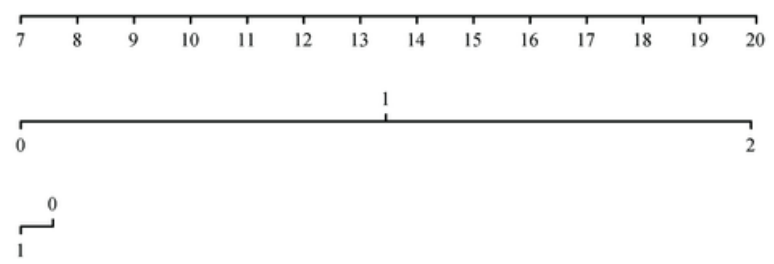

Radiotherapy

0
1

TMZ chemotherapy

IDH Status

Total Points

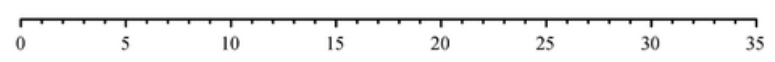

1-year survival

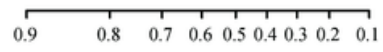

3-year survival

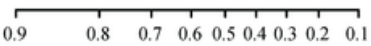

5-year survival

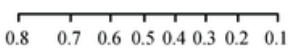

\section{Figure 6}

Nomogram for predicting the survival rate of glioma patient. (A) AUCs for predicting CNTNAP3, mTOR, NFATC3, NOM10S, and their interaction were $0.572,0.590,0.623,0.659$ and 0.687 , respectively. (B) survival plot of the prognostic risk model. (C) A nomogram was established based on the risk signature, age, glioma type (0: primary glioma, 2: secondary glioma, and 1: recurrent glioma), gender (0: male, 1: female), radiotherapy (0:No, 1: Yes), TMZ chenmotherapy (0:No, 1: Yes), and IDH status (1: mutuant, 0 : wild-type) for predicting survival of glioma patient. 


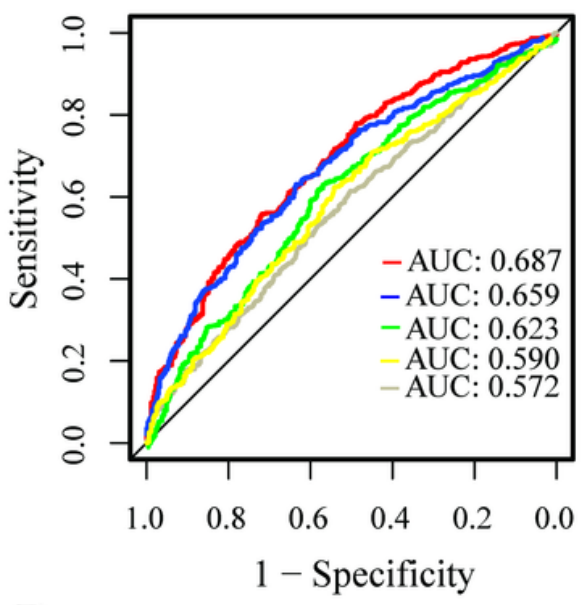

B

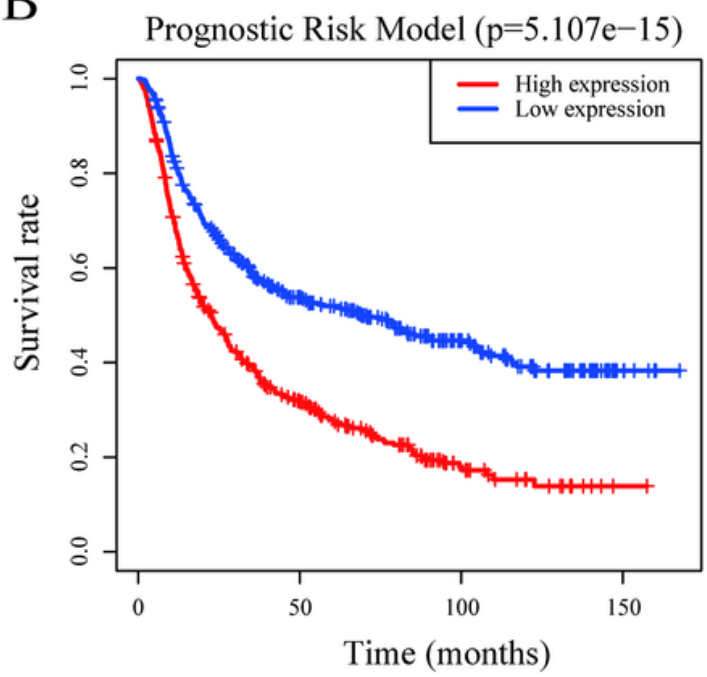

Points

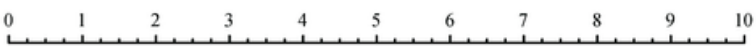

NOM1
CNTNAP3 Age

Risk Score

Glioma type

Gender
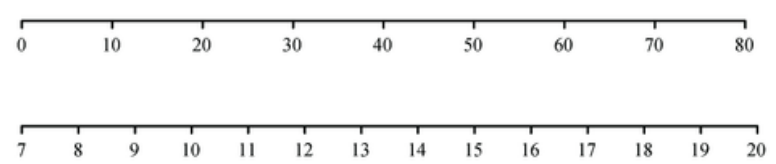

Radiotherapy

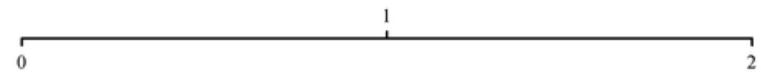

$\overbrace{}^{0}$

TMZ chemotherapy

IDH Status

Total Points

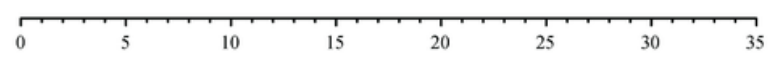

1-year survival

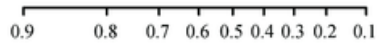

3-year survival

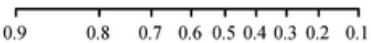

5-year survival

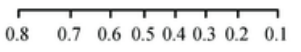

\section{Figure 6}

Nomogram for predicting the survival rate of glioma patient. (A) AUCs for predicting CNTNAP3, mTOR, NFATC3, NOM10S, and their interaction were $0.572,0.590,0.623,0.659$ and 0.687 , respectively. (B) survival plot of the prognostic risk model. (C) A nomogram was established based on the risk signature, age, glioma type (0: primary glioma, 2: secondary glioma, and 1: recurrent glioma), gender (0: male, 1: female), radiotherapy (0:No, 1: Yes), TMZ chenmotherapy (0:No, 1: Yes), and IDH status (1: mutuant, 0 : wild-type) for predicting survival of glioma patient.

\section{Supplementary Files}

This is a list of supplementary files associated with this preprint. Click to download.

- SupplementaryFigure1.tif

- SupplementaryFigure1.tif 